IOSR Journal of Pharmacy

e-ISSN: 2250-3013, p-ISSN: 2319-4219, www.iosrphr.org

Volume 2 Issue 6 || || Nov-Dec. 2012 || || PP.01-09

\title{
Effects of D-002, a mixture of beeswax alcohols, on osteoarthritis symptoms: a randomized placebo-controlled study
}

\author{
Ivan Rodríguez, ${ }^{1}$ Sarahí Mendoza, ${ }^{2}$ José Illnait, ${ }^{1}$ Rosa Mas, ${ }^{2}$ Julio César \\ Fernández, ${ }^{2}$ Lilia Fernández, ${ }^{2}$ Meilis Mesa, ${ }^{1}$ Rafael Gámez ${ }^{2}$ \\ A concise title: D-002 on osteoarthritis symptoms \\ ${ }^{1}$ Surgical Medical Research Centre (Havana, Cuba) \\ ${ }^{2}$ Centre of Natural Products, National Centre for Scientific Research (Havana, Cuba) \\ Disclosure: No potential conflicts of financial interest relevant to this article were reported.
}

\begin{abstract}
Background and purpose: Non selective non-steroidal anti-inflammatory drugs and ciclooxygenase 2 inhibitors provide symptoms relief in osteoarthritis $(\mathrm{OA})$, but produce several adverse effects (AE), which supports the search for other options. D-002, a mixture of higher aliphatic beeswax alcohols, has been shown to produce anti-inflammatory effects experimentally. This double-blind study investigated the effects of $D$ $002(50 \mathrm{mg} /$ day $)$ on patients with OA symptoms randomized to D-002 $(50 \mathrm{mg})$ or placebo for 8 weeks.

Methods: The primary efficacy outcome was the reduction of the total Western Ontario and McMaster Individual Osteoarthritis Index (WOMAC) score. The decrease on pain, joint stiffness, physical activity scores, and use of rescue medications were secondary outcomes. Treatment tolerability was assessed.

Results: Four patients (3 D-002, 1 placebo) discontinued the study, none due to adverse experiences $(A E)$. At study completion D-002 significantly reduced the total WOMAC score versus baseline $(p<0.0001$, 49.9\%) and placebo ( $p<0.001,38.0 \%)$, and also decreased the pain ( $p<0.001 ; 30.6 \%)$, joint stiffness ( $p<0.05$ $26.5 \%)$ and physical activity ( $p<0.0001,41.2 \%)$ scores versus placebo. After 2 weeks on therapy all benefits were significant already. The use of rescue medication in D-002 (3/30, 10\%) was lower $(p<0.05)$ than in placebo (10/30; 33.3\%). Treatment was well tolerated. Five patients (2 D-002, 3 placebo) reported mild $A E$.

Conclusions: D-002 (50 mg/day) given for 8 weeks improved $O A$ symptoms, the benefits being evident from week 2, and was well tolerated. D-002 could be a suitable option for managing OA symptoms, but this appreciation requires extensive further research.
\end{abstract}

Keywords-anti-inflammatory, beeswax alcohols, D-002, osteoarthritis, WOMAC score

\section{INTRODUCTION}

Osteoarthritis (OA), a painful and disabling joint condition that affects hundreds of millions worldwide, mainly the elderly[1], requires both non-pharmacological [2, 3] and pharmacological interventions [4-7]. Current guidelines recommend use analgesics or non-steroidal anti-inflammatory drugs (NSAIDs) to provide symptoms relief despite they do not solve the underlying causal pathological process [4-7]. Nevertheless, in view of the gastrointestinal and cardiovascular adverse effects (AE) of non selective NSAIDs and specific ciclooxygenase 2 (COX-2), respectively $[8,9]$, the search for better tolerated alternatives is justified.

D-002 is a mixture of six higher aliphatic primary alcohols $\left(\mathrm{C}_{26}, \mathrm{C}_{26}, \mathrm{C}_{28}, \mathrm{C}_{30}, \mathrm{C}_{32}, \mathrm{C}_{34}\right)$ purified from the beeswax, wherein triacontanol $\left(\mathrm{C}_{30}\right)$ is the most abundant, with anti-inflammatory effects demonstrated in experimental models of acute and chronic inflammation [10-12]. Oral treatment with D-002, however, is gastroprotective, rather than gastrotoxic, through a mechanism that involves increased secretion and improved composition of the gastric mucus, and reduced lipid peroxidation in the gastric mucosa [14-16]. Antioxidant effects of D-002 have been demonstrated in experimental and clinical studies [21-27]. Keeping in mind this background, we believed that D-002 could improve OA symptoms.

\section{OBJECTIVE}

This study investigated the effects of D-002 (50 mg/day) on patients with symptoms of OA 


\section{METHODS}

3.1 Study design

This randomized, double-blind, placebo-controlled trial was approved by the Institutional Ethics Committee of the Surgical Research Centre (Havana, Cuba) and was conducted in compliance with the ethical standards for the treatment of patients as established in the Declaration of Helsinki.

Patients were provided oral and written explanations about the nature of the trial and the study treatment in a language easily understood by the subjects in order to request their informed written consent, which was obtained from each of the participants at enrolment (visit 1).

Eligible patients were randomised to D-002 $(50 \mathrm{mg}$ ) or placebo (visit 2) tablets, which should be taken once a day with the breakfast for 8 weeks. Subjects underwent to visits every 2 weeks (visits 3 - 6). Physical examinations and assessment of WOMAC scores were done at each visit. Treatment compliance, rescue medication (analgesics) consumption and $\mathrm{AE}$ were controlled from visit 3 to 6 , laboratory examinations were done at baseline and every 4 weeks.

\subsection{Study participants}

Ambulatory women and men of 20 - 80 years of age, with a previous diagnosis of OA of the knee, hip or fingers supported by clinical and radiological criteria, were enrolled in the trial. Participants were required to have a diagnosis of functional class I, II or III (mild to moderate) in accordance to the American College of Rheumatology Criteria (ACRC) [28, 29].

Exclusion criteria were to suffer other forms of arthritis, any arthroscopy within the past year, intra-articular injection of steroids within the past 3 months, uncontrolled hypertension (diastolic pressure $\geq 120 \mathrm{~mm} \mathrm{Hg}$ ) or diabetes (fasting glucose $>7 \mathrm{mmol} / \mathrm{L}$ ), active liver or renal disease, malignancies, or any other serious illnesses. Also, we excluded pregnant or lactating women, or those not taking adequate contraceptive measures and subjects with the following laboratory abnormalities: alanine -ALT- and/or aspartate amino tranferase-AST $>45$ $\mathrm{U} / \mathrm{L}$, creatinine $>130 \mu \mathrm{mol} / \mathrm{L}$, and/or those with any hospitalization during the 6 months prior to the study. Unwillingness to follow-up, to experience AE that justified such decision and protocol violations (failure of treatment intake $\geq 5$ days) were predefined causes of premature discontinuations of the study.

\subsection{Treatment}

Study treatments, produced under Licensees and Good Manufacturing Practices conditions, came from the manufacturers (Plants of Natural Products, Laboratorios MedSol, Havana, Cuba). D-002 content was assessed by using a gas chromatography method [30]. Placebo had similar composition to D-002 tablets, except the active ingredient that was replaced by lactose. Treatments were packaged in PVC-aluminium sealed burbles (blisters).

At visit 2, identical coded and packaged tablets of study treatments (D-002 $50 \mathrm{mg}$ or placebo) were given to study subjects. Tables were taken once a day with the breakfast for 8 weeks. No dose titration was done. The randomisation code was computer-generated with a fixed, not stratified randomisation method, using balanced blocks and allocation ratio of $1: 1$. The dose of D-002 $(50 \mathrm{mg} / \mathrm{day})$ selected had been shown to produce effective antioxidant effects in clinical studies [24-27].

The entire code was kept confidential at the generating place. Sealed individual envelopes with codes of each subject were kept at the generating place and at the site of the Principal Investigator, which should be opened prematurely in case of occurring a serious adverse event (SAE), a situation that did not occur in the trial. Treatment compliance was controlled by counting the remainder tablets and making interviews to subjects. At trial completion, non-used tablets were recovered. Compliance was considered good if the subjects consumed at least $85 \%$ of the tablets scheduled from the previous visit.

Subjects were not allowed to consume NSAIDs, steroids, cartilage or calcium supplements, or any other agent that may affect the study outcomes, except the rescue medications to treat persistent pain: acetaminophen (maximum $2 \mathrm{~g} / \mathrm{day}$ ) or metamizole (maximum $600 \mathrm{mg} / \mathrm{day}$ ). The number of consumed rescue medication tablets was recorded at each visit.

\subsection{Efficacy Assessment}

The primary end-point was to obtain a significant reduction of the total Western Ontario and McMaster Individual Osteoarthritis Composite (WOMAC) index [31, 32] (Table 1) of not less than 30\% as compared to placebo. At each visit, subjects completed the WOMAC questionnaire, which consists of three sections, one that assess pain intensity (5 questions), other joint stiffness ( 2 questions), and the third the physical function (17 questions). Individual responses were scored on the following scale: 0 (none), 1 (slight), 2 (moderate), 3 (severe) and 4 (extreme). The total score ranges from 0 (the best) to 96 (the worst).

Reductions in pain, stiffness and physical function scores were secondary efficacy variables. For efficacy, the score reductions should be significant as compared to placebo. 
Use of rescue medication was addressed as a collateral measure of efficacy. The amount of rescue medication was assessed in terms of total use at the conclusion of the study period.

\subsection{Safety and tolerability assessment}

The safety indicators included vital signs (body weight, pulse rate, blood diastolic and systolic pressure), and blood indicators (erythrocyte sedimentation rate, ALT, AST, serum fasting glucose and creatinine). Blood biochemical safety indicators were assessed with enzymatic methods by using reagent kits (Roche, Switzerland) and performed in the Hitachi 709 autoanalyser (Tokyo, Japan), erythrocyte sedimentation rate was assessed by conventional method, all done at the clinical laboratory of the Surgical and Medical Research Centre (Havana, Cuba). Controls of the precision and accuracy of the methods were performed.

We considered as AEs all undesirable events that occurred to a subject during the study, disregarding the cause, whenever they newly appeared during the trial. Subjects were queried by investigators for any AEs between study visits. AEs were recorded in the case record forms, including their characteristics, dates of onset and disappearance, treatments adopted and responses achieved. Severity of AEs was classified as mild, moderate or serious (SAE), mild being those easily tolerated that not required suspension of study medications and/or specific treatment, moderate those that caused discomfort enough and required stopping therapy and/or specific treatment, and SAE those disabling events that leaded to hospitalisation and/or deaths, if happened. AEs that occurred within 30 days of consuming the last study doses, monitored by direct contact with the subjects, were included in this analysis. The causal relationships between AEs and the treatments were classified by using the Naranjo algorithm [33].

\subsection{Statistical Analysis}

Data were analysed as per the intention to treat (ITT) approach. So, data of all randomized subjects were included in all analyses. The sample size estimation assumed a difference of $\geq 30 \%$ between the reduction of WOMAC total scores from baseline with D-002 and placebo at study completion. Then, 30 subjects per treatment arm would be sufficient to detect such difference with $80 \%$ power and $\alpha=0.05$. Assuming a permissible dropout rate of $10 \%, 65$ subjects were enrolled.

Continuous data were analyzed by using the following tests: unpaired and paired $t$ tests, Bonferroni adjustment for multiple comparisons, or ANOVA, as appropriate. Categorical variables were compared with the Fisher Exact Probability test. All statistical tests for differences were 2-tailed. The following software was used for the comparisons: Statistics software for Windows (USA) and MS Excel. Statistical significance was taken at the 95\% level $(\mathrm{p}<0.05)$.

\subsection{Baseline characteristics}

\section{RESULTS}

Sixty-five (65) subjects were enrolled in the study. Of them, 60 were eligible for randomization. Five enrolled subjects did not pass to the active treatment step because of the following reasons: fasting glucose $>7$ $\mathrm{mmol} / \mathrm{L}$ ( 2 subjects), AST and ALT values $>55 \mathrm{U} / \mathrm{L}$ ( 2 cases) and rheumatoid arthritis (1). Of the 60 patients (50 women, 10 men) (mean age 52 years) included, 56 completed the trial and 4 (3 D-002, 1 placebo) withdrew from the study, none due to AE.

All baseline characteristics of both groups were similar, so that subject randomization was effective (Table 2). Gender was predominantly female 50/60 (83.3\%) vs. males 10/60 (16.7\%). Study population included a high frequency (>30\%) of some co-morbid conditions like hypertension (41.7\%) and hypercholesterolemia (36.7\%), and some negative lifestyle factors, like sedentary life (73.3\%) and smoking (20.0\%). A total of 45/60 (75\%) randomized subjects consumed some concomitant therapy during the study.

Four subjects (3 D-002, 1 placebo) withdrew prematurely from the study: 2 due to protocol violations (D-002) and 2 due to unwillingness to follow-up (1 D-002, 1 placebo).

\subsection{Efficacy analysis}

Treatment compliance was very good and similar in both groups.

At baseline the total WOMAC scores (mean \pm SD) in the D-002 and placebo groups were $41.5 \pm 13.0$ and 42.1 \pm 14.9 , respectively, without significant differences between the groups (Table 2). After 2 weeks of treatment, the score was significantly reduced in D-002 ( $p<0.001$ versus baseline) and placebo ( $p<0.05$ versus baseline) by 30.8 and $8.8 \%$, respectively, but the total WOMAC score in D-002 group was lower $(\mathrm{p}<0.05)$ than in placebo (net difference of $22 \%$ versus placebo). The treatment effect did not wear off, but even improved, during the treatment period. At study completion D-002 had reduced significantly the total WOMAC score ( $p$ $<0.0001$ versus baseline and placebo) (49.9\% versus baseline, $38.0 \%$ versus placebo)

The mean \pm SD baseline WOMAC pain scores were $10.5 \pm 3.5$ (D-002 group) and $9.9 \pm 4.0$ (placebo) (Table 4). After 2 weeks of treatment, pain score was significant lower in the D-002 (28.6\% reduction versus 
baseline, $\mathrm{p}<0.001)$ and placebo $(11.1 \%$ reduction versus baseline, $\mathrm{p}<0.05)$ groups, so that the net difference versus placebo was $17.5 \%$. The treatment effect was enhanced over the trial, so that at the study completion pain score decreased significantly with D-002 (45.7\% versus baseline, $\mathrm{p}<0.0001) 30.6 \%, \mathrm{p}<0.01$ versus placebo).

At baseline the mean stiffness score was $3.4 \pm 2.1$ in the D-002 group, and $3.4 \pm 1.8$ in placebo. After week 2, the score was significantly reduced with D-002 (32.3\% versus baseline, $\mathrm{p}<0.001 ; 23.5 \%$ versus placebo, $\mathrm{p}$ $<0.01$ ). At study completion the reduction in stiffness with D-002 ( $p<0.01$ versus baseline, $p<0.05$ versus placebo) was of $41.2 \%$ as compared to baseline, and of $26.5 \%$ versus placebo.

The sequential changes in WOMAC physical function scores were similar to those occurred with the other subset and total WOMAC scores. Meanwhile the mean baseline values of D-002 (27.5 \pm 9.6$)$ and placebo $(28.7 \pm 10.8)$ groups were similar, the scores at 2,4 and 8 weeks of therapy in the D-002 group were significantly lower ( $<<0.001$ for week $2, \mathrm{p}<0.0001$ for weeks 4 and 8 ) than in placebo. The score reductions with D-002 were successively accentuated over the 8 week period, so that the decrease of the physical function score with D-002 at trial completion was of $51.3 \%$ and $41.2 \%$ as compared to baseline and placebo, respectively.

Thirteen (13) subjects (3 D-002, 10\%; 10 placebo, 33.3\%) consumed acetaminophen or metamizole during the trial, so that the consumption of rescue medication in D-002 group was lower $(\mathrm{p}<0.05)$ than in placebo group.

\subsection{Safety and tolerability}

Vital signs and blood indicators did not change during the study, with the exception of erythrocyte sedimentation rate, which significantly lowered from baseline in D-002 group as compared to baseline ( $\mathrm{p}$ $<0.0001)$ and placebo $(\mathrm{p}<0.05)$ (data not shown for simplicity). This reduction, albeit significant, was not considered as an $\mathrm{AE}$.

\section{DISCUSSION}

Using a randomized, double-blind placebo-controlled design the present study demonstrated, by the first time, that D-002 improved pain, stiffness, physical function and overall activity in subjects with OA symptoms as compared to baseline and placebo. The D-002 effects were persistent over the trial, so that it produced sustained benefits, distinguishable from placebo.

D-002 and placebo groups were homogeneous at baseline, which indicates that randomization was effective, and that our results are not attributable to initial differences between the groups, but to D-002 treatment. The mean age of study subjects (52 years) falls within that expected for this disease. Most subjects (83.3\%) were women, consistent with a higher prevalence of OA in women, mainly post-menopausal [34], a condition present in 31 of the 50 randomized women (62\%). The high frequency of sedentary life (81.7\%), hypertension (41.7\%), hypercholesterolemia (36.7\%) and smoking (20\%) among study subjects, not only reflects an undesirable occurrence of coronary risk factors, common in Cuban subjects of this age [35], but agrees with reports of co-morbidity of OA in middle-aged and older subjects [36].

Both groups displayed an improvement of total WOMAC values over the 8 weeks of treatment, including placebo, but these reductions, however, were greater in D-002 than in placebo group. A similar picture was seen for subset WOMAC scores. The D-002 group significant reductions of the total (primary efficacy variable) and subset (secondary efficacy variables) WOMAC scores were evident from the week 2, with appreciable improvements with continued administration. At study completion pain, stiffness, physical function and total WOMAC scores decreased by $45.7 \%, 41.2 \%, 51.3 \%$ and $49.9 \%$ as compared to baseline, respectively (reductions versus placebo of $30.6 \%, 26.5 \%, 41.2 \%$, and $38.0 \%$, respectively).

In contrast, although reductions with placebo were also seen, they remained almost stationary over the treatment. The final decreases of pain, stiffness, physical function and total WOMAC scores in placebo were $15.1 \%, 14.7 \%, 10.1 \%$ and $11.9 \%$, respectively, versus baseline. This improvement with placebo, however, was not totally unexpected, as it can occur in any efficacy measurement based on subjective assessment. Possible explanations for this finding could include that study subjects may have had high expectations of the benefits of study treatments, despite they were due informed about the use of placebo, and that $33.3 \%$ of placebo subjects used rescue medications over the trial, so that the reductions observed in the placebo group could be influenced by this fact, as in other placebo-controlled studies in subjects with OA [37].

We believe that the placebo effect here seen, however, does not limit the acceptance of the D-002 efficacy for alleviating OA symptoms here demonstrated. The evident significance of the score reductions with D-002 as compared to placebo and the fact that, opposite to the increasingly efficacy of D-002, the magnitude of the placebo effect was steady over the trial support this appreciation. Besides, since the use of rescue medication use in placebo was lower than in D-002 group, it becomes obvious that the efficacy results with D-002 are actually due to the treatment. In addition, this fact minimizes the possible contribution of a masking effect of rescue medication to efficacy results, since despite to be more frequently used in placebo, all reductions, even for pain subset, were more effective in D-002 group. 
Although the detailed mechanisms by which D-002 achieves reductions of OA symptoms remain unclear, they should be associated with its anti-inflammatory [11, 12], and moderate analgesic effects [12]. D-002 has been shown to reduce leukotriene B4 content in the pleural exudates of rats with carrageenan-induced pleurisy [11], and myeloperoxidase activity in mice with xylene-induced ear oedema [12], which suggest that its effects on lipooxygenase (5-LOX) pathway and neutrophil infiltration, respectively, could be associated with its anti-inflammatory effects in vivo. Currently, the effects of D-002 on both COX and 5-LOX activities are under study.

Some nutraceuticals with anti-inflammatory effects and good gastrointestinal safety profile have shown benefits in experimental arthritis and in OA patient, as well [38]. Since COX-2 is intimately involved in OA symptoms, dietary n-3 polyunsaturated fatty acids (PUFA), which inhibit COX activity, are thought to be beneficial for managing arthritis, and given together with glucosamine sulfate, has provided a superior efficacy as compared to glucosamine alone in patients with OA [39, 40].

D-002 resulted safe and well tolerated, consistent with previous clinical studies [18-20, 24-27]. In particular, the absence of gastrointestinal AEs matches well with the gastroprotective effects of D-002 [18-20]. The existing concern about the increased risk for cardiovascular disease and stroke with COX-2 inhibitors, and the gastrointestinal and renal complications produced by non-selective NSAIDs [7-9], remains open perspectives for new therapies, including complementary medicines. Indeed, OA sufferers using NSAIDs have an increased risk of suffering AEs that require hospitalization as compared to the non-users [9]. Then, these subjects look to other alternatives for symptomatic relief devoid of relevant AEs [38].

There are a number of limitations of the current study that require further research. The number of study participants is small, and we did not confirm the previous diagnosis of OA. Then, although the reductions of all WOMAC scores with D-002 as compared to placebo suggest that this treatment, devoid of gastrotoxic effects, could be useful to manage OA symptoms, the efficacy of D-002 needs to be adequately determined in further clinical trials to support this preliminary concept.

\section{CONCLUSION}

D-002 treatment $(50 \mathrm{mg} / \mathrm{day})$ for 8 weeks improved OA symptoms as compared to placebo. The benefits were evident from week 2 . Treatment tolerability was very good. Then, D-002 could be a suitable option for managing OA symptoms, but this appreciation requires extensive further research.

\section{REFERENCES}

[1]. C. Lozada, and E. Steigelfest, Osteoarthritis, Medicine Rheumatology, 15, 2010, 12-23.

[2]. E. Roddy, and M. Doherty, Changing life-styles and osteoarthritis: what is the evidence?, Best Pract Res Clin Reumatol, 20, 2006, 81-97.

[3]. YC. Lee, and RH.Shmerling, The benefit of non-pharmacologic therapy to treat symptomatic osteoarthritis, Curr Rheumatol Rep, 10, 2008, 5-10.

[4]. B. Bannwarth, Acetaminophen or NSAIDs for the treatment of osteoarthritis, Best Pract Res Clin Rheumatol, 20, 2006, 117-29.

[5]. TE. Towheed, L. Maxwell, M. Judd, M. Catton, MC. Hochberg, and G. Wells, Acetaminophen for osteoarthritis, Cochrane Database Syst Rev, 1, 2006, CD004257.

[6]. W. Zhang, R. Moskowitz, G. Nuki, S. Abramson, RD. Altman, N. Arden, et al, OARSI recommendations for the management of hip and knee osteoarthritis, Part II: OARSI evidence-based, expert consensus guidelines, Osteoarthritis Cartilage, 16, 2008,137-62.

[7]. CR. Scanzello, and NK. Moskowitz, The post-NSAID era: what to use now for the pharmacologic treatment of pain and inflammation in osteoarthritis, Curr Rheumatol Rep, 10, 2008, 49-56.

[8]. Dickman, and J. Ellershaw, NSAIDs: gastroprotection or selective COX-2 inhibitor?, Palliat Med, 18, 2004, 27586.

[9]. RA. Moore, S. Derry, and HJ. McQuay, Cyclo-oxygenase-2 selective inhibitors and non-steroidal antiinflammatory drugs: balancing gastrointestinal and cardiovascular risk, BMC Musculoskelet Disord, 8, 2007, $73-7$.

[10]. R. Más, D-002: A product obtained from beeswax, Drugs of the Future, 26, 2001, 731-44.

[11]. D. Carbajal, V. Molina, S. Valdés, ML. Arruzazabala, and R. Mas, Anti-inflammatory activity of D-002: an active product isolated from beeswax, Prostagl Leukotr Essent Fatty Acids, 59, 1998, 235-8.

[12]. Y. Ravelo, V. Molina, D. Carbajal, ML. Arruzazabala, R. Mas, A. Oyarzábal, et al, Evaluation of antiinflammatory and antinociceptive effects of Abexol (beeswax alcohols), J Nat Med, 65, 2010, 330-5.

[13]. D. Carbajal, V. Molina, S. Valdés, ML. Arruzazabala, and R. Mas, Anti-ulcer activity of higher primary alcohols of beeswax, J Pharm Phamacol, 47, 1995, 731-3.

[14]. D. Carbajal, V. Molina, S. Valdés, ML. Arruzazabala, I. Rodeiro, R. Mas, et al, Possible cytoprotective mechanism in rats of D-002 an anti-ulcerogenic product isolated from beeswax, J Pharm Pharmacol, 48, 1996, 858-60.

[15]. D. Carbajal, V. Molina, M. Noa, S. Valdes, ML. Arruzazabala, A. Aguiar, et al, Effects of D-002 on gastric mucus composition in ethanol-induced ulcer, Pharmacol Res, 42, 2000, 329-32.

[16]. V. Molina, S. Valdés, D. Carbajal, ML. Arruzazabala, R. Menéndez, and R. Mas, Antioxidant effects of D-002 on gastric mucosa of rats with experimentally-induced injury, J Med Food, 4, 2001, 79-83. 
[17]. V. Molina, D. Carbajal, ML. Arruzazabala, and R. Mas, Therapeutic effect of D-002 (Abexol) on gastric ulcer induced experimentally in rats, J Med Food, 18, 2005, 59-62.

[18]. O. Hano, J. Illnait, R. Mas, L. Fernández, F. Piñol, and J. Fernández, Effects of D-002, a product isolated from beeswax, on duodenal ulcer: a double-blind, placebo-controlled study, Curr Ther Res, 62, 2001, 394-407.

[19]. J. Illnait, H. Terry, R. Mas, L. Fernandez, D. Carbajal, Fernández JC, et al, Effects of D-002, a product isolated from beeswax, on gastric symptoms of patients with osteoarthritis treated with piroxicam: a pilot study, J Med Food, 8, 2005, 63-8.

[20]. Rodríguez, J. Illnait, H. Terry, R. Más, L. Fernandez, JC. Fernández, et al, Effects of Abexol ® (beeswax alcohols) on gastrointestinal symptoms of middle-aged and older subjects, Rev CENIC Cien Biol, 40, 2009, 147-54.

[21]. R. Menéndez, AM. Amor, RM. González, S. Jiménez, and R. Mas, Inhibition of rat microsomal lipid peroxidation by the oral administration of Abexol, Brazil J Med Biol Res, 33, 2000, 85-90.

[22]. V. Molina, S. Valdés, D. Carbajal, ML. Arruzazabala, R. Menendez, and R. Más. Antioxidant effects of Abexol on gastric mucosa of rats with injury induced experimentall, J Med Food, 4, 2001,79-84.

[23]. S. Mendoza, M. Noa, Y. Perez, and R. Mas, Preventive effect of Abexol, a mixture of long-chain alcohols from beeswax, on the liver damage induced with CCl4 in rats, J Med Food, 10, 2007,379-83.

[24]. R. Menéndez, R. Mas, AM. Amor, Y. Pérez, RM. González, JC. Fernández, et al, Antioxidant effects of Abexol on the in vitro susceptibility in healthy volunteers, Arch Med Res, 32, 2001, 436-41.

[25]. R. Menéndez, R. Mas, J. Illnait, Y. Pérez, AM. Amor, JC. Fernández, et al, Effects of Abexol on lipid peroxidation in older subjects, J Med Food, 4, 2001, 71-7.

[26]. E. López, J. Illnait, V. Molina, A. Oyarzábal, L. Fernández, Y. Pérez, et al, Effects of Abexol (beeswax alcohols) on lipid peroxidation in middle-aged and older subjects, Lat Am J Pharm, 27, 2008, 695-703.

[27]. Rodriguez, J. Illnait, V. Molina, A. Oyarzábal, L. Fernández, et al, Comparison of the antioxidant effects of D-002 (beeswax alcohols) and grape seed extract (GSE) on plasma oxidative variables in healthy subjects, Lat Am J Pharm, 29, 2010, 255-62.

[28]. R. Altman, E. Asch, D. Bloch, G. Bole, and D. Bronestein, The American College of Rheumatology Criteria for the classification and reporting of osteoarthritis of the knee, Arthritis Rheum, 9, 1986, 1039-49.

[29]. R. Altman, G. Alarcón, D. Appelrouth, D. Bloch, D. Borenstein, and K. Brandt, The American College of Rheumatology Criteria for the classification and reporting of osteoarthritis of the hip, Arthritis Rheum, 34, 1991, $505-14$.

[30]. V. González, D. Marrero, R. Sierra, C. Velázquez, and R. Vicenre, Nuevo método por Cromatografía Gaseosa Capilar para el análisis del ingrediente activo D002, Rev CENIC Cien Quím, 39, 2008, 123-4.

[31]. E. Batlle, J. Esteve, MC. Piera, R. Hargreaves, and J. Cutts, Adaptación transcultural al cuestionario WOMAC especifico para artrosis de rodilla y cadera, Rev Esp Reumatol, 26,1999, 38-45.

[32]. T. Angst, A. Aeschlimann, and G. Stuki, Smallest detectable and minimal clinically important differences of rehabilitation intervention with their implication for required simple sizes using WOMAC and SF-36 quality of life measurement instruments in patients with osteoarthritis of the lower extremities, Arthritis and Rheumatism, 45, 2001, 384-91.

[33]. CA. Naranjo, U. Busto, EM. Sellers, P. Sandor, I. Ruiz, EA. Roberts, et al, A method for estimating the probability of adverse drug reactions, Clin Pharmacol Ther, 30, 1981, 239-45.

[34]. G. Peat, R. McCarney, and P. Croft, Knee pain and osteoarthritis in older adults: Review of community burden and current use of primary health care, Ann Rheum Dis, 60, 2001, 91-7.

[35]. Cuban Health Ministry, National Direction of Medical Register and Health Statistics, Health Annual Report 2010, http://www.one.cu/publicaciones/03estadisticassociales/Salud\%20en\%20Cifras\%20Cuba\%202010/edo.pdf. Accessed 19 May 2011.

[36]. G. Singh, D. Miller, FH. Lee, D. Pettitt, and MW. Russell, Prevalence of cardiovascular disease risk factors among US adults with self-reported osteoarthritis: data from the Third National Health and Nutrition Examination Survey, Am J Manag Care, 8, 2002, S428-30.

[37]. DO. Clegg, DJ. Reda, CL. Harris, MA. Klein, JR. O'Dell, MM. Hooper, et al, Glucosamine, chondroitin sulfate, and the two in combination for painful knee osteoarthritis, N Engl J Med, 354, 2006,795-808.

[38]. TM. Frech, and DO. Clegg, The utility of nutraceuticals in the treatment of osteoarthritis, Current Rheumatol Reports, 9, 2010, 25-30.

[39]. S. Hurst, SG. Rees , PF. Randerson , B. Caterson, and JL. Harwood, Contrasting effects of n-3 and n-6 fatty acids on cyclooxygenase-2 in model systems for arthritis, Lipids, 44, 2009, 889-96.

[40]. J. Gruenwald, E. Petzold, R. Busch, HP. Petzold , and HJ. Graubaum, Effect of glucosamine sulfate with or without omega-3 fatty acids in patients with osteoarthritis, Adv Ther, 26, 2009, 858-71. 
Table 1 Modified WOMAC Questionnaire.

\begin{tabular}{|l|}
\hline WOMAC pain assessment at \\
\hline Walking \\
Stair climbing \\
Night \\
Rest \\
Weight bearing \\
\hline WOMAC Stiffness assessment \\
\hline In morning \\
Occurring during the day \\
\hline WOMAC Physical function assessment (difficulty for) \\
\hline Descending stairs \\
Ascending stairs \\
Rising from sitting \\
Standing \\
Bending to the floor \\
Walking on flat \\
Getting in/out of a car \\
Going shopping \\
Putting on socks-Rising from bed \\
Taking off socks \\
Lying in bed \\
Getting in/out of bath \\
Sitting \\
Getting on/off toilet \\
Heavy domestic duties \\
Light domestic duties \\
While working \\
Sitting cross legged \\
While cycling \\
While driving vehicle \\
While praying \\
\hline
\end{tabular}

Responses of subjects corresponded to the following score:

$0=$ none $; 1=$ slight $; 2=$ moderate $; 3=$ severe $;=$ extreme 
Effects of D-002, a mixture of beeswax alcohols, on osteoarthritis symptoms...

Table 2. Baseline demographic and clinical characteristics of study population

\begin{tabular}{|c|c|c|c|c|c|c|}
\hline Characteristic & \multicolumn{2}{|c|}{ D-002 $(n=30)$} & \multicolumn{2}{|c|}{ Placebo $(\mathbf{n}=30)$} & \multicolumn{2}{|c|}{ Total $(n=60)$} \\
\hline \multicolumn{7}{|l|}{ Age (years) } \\
\hline Mean \pm SD & \multicolumn{2}{|c|}{$52 \pm 10$} & \multicolumn{2}{|c|}{$53 \pm 10$} & \multicolumn{2}{|c|}{$52 \pm 10$} \\
\hline Range & \multicolumn{2}{|c|}{$31-71$} & \multicolumn{2}{|c|}{$34-72$} & \multicolumn{2}{|c|}{$31-72$} \\
\hline Body mass index $\left(\mathrm{kg} / \mathrm{m}^{2}\right)($ Mean $\pm \mathrm{SD})$ & \multicolumn{2}{|c|}{$27.2 \pm 4.3$} & \multicolumn{2}{|c|}{$26.5 \pm 4.4$} & \multicolumn{2}{|c|}{$26.9 \pm 4.3$} \\
\hline Total WOMAC scores (Mean \pm SD) & \multicolumn{2}{|c|}{$41.5 \pm 13.0$} & \multicolumn{2}{|c|}{$42.1 \pm 14.9$} & \multicolumn{2}{|c|}{$41.8 \pm 13.8$} \\
\hline Sex & $\mathbf{n}$ & $\%$ & $\mathbf{n}$ & $\%$ & $\mathbf{n}$ & $\%$ \\
\hline Female & 26 & 86.7 & 24 & 80.0 & 50 & 83.3 \\
\hline Male & 4 & 13.3 & 6 & 20.0 & 10 & 16.7 \\
\hline Subjects with previous NSAID therapy & 17 & 56.7 & 15 & 50.0 & 32 & 53.3 \\
\hline \multicolumn{7}{|l|}{ Main concomitant conditions } \\
\hline Hypertension & 12 & 40.0 & 13 & 43.3 & 25 & 41.7 \\
\hline Hypercholesterolemia & 11 & 36.7 & 11 & 36.7 & 22 & 36.7 \\
\hline Thyroid dysfunction & 3 & 10.0 & 3 & 10.0 & 6 & 10.0 \\
\hline Diabetes & 1 & 3.3 & 1 & 3.3 & 2 & 3.3 \\
\hline \multicolumn{7}{|l|}{ Lifestyle factors } \\
\hline Sedentary life & 24 & 80.0 & 25 & 83.3 & 49 & 81.7 \\
\hline Smoking & 5 & 16.6 & 7 & 23.3 & 12 & 20.0 \\
\hline \multicolumn{7}{|l|}{ Concomitant therapy $^{\text {a }}$} \\
\hline Consumption of al least one concomitant & 23 & 76.7 & 22 & 73.3 & 45 & 75.0 \\
\hline Diuretics & 9 & 30.0 & 10 & 33.3 & 19 & 31.7 \\
\hline Cholesterol-lowering drugs & 10 & 33.3 & 9 & 30.0 & 19 & 31.7 \\
\hline Angiotensin converting enzyme inhibitors & 6 & 20.0 & 6 & 20.0 & 12 & 20.0 \\
\hline Thyroid hormones & 3 & 10.0 & 3 & 10.0 & 6 & 10.0 \\
\hline$\beta$-blockers & 2 & 6.7 & 3 & 10.0 & 5 & 8.3 \\
\hline Calcium antagonists & 1 & 3.3 & 2 & 6.7 & 3 & 5.0 \\
\hline
\end{tabular}

SD: standard deviation, $\mathrm{n}$ : number of cases , NSAID: non steroidal anti-inflammatory drugs, OA: osteoarthritis

a The table includes only those consumed by $\geq 2$ subjects

No significant between group differences were found, ( $t$ test for independent samples for continuous variables, Fisher Exact Probability test for categorical variables)

Table 3. Changes in Total Western Ontario and McMaster Individual Osteoarthritis Index (WOMAC) scores

\begin{tabular}{|l|c|c|}
\multicolumn{2}{|c|}{ WOMAC Index scores } \\
\hline Baseline & $\mathbf{D - 0 0 2}$ & Placebo \\
2 Weeks & $41.5 \pm 13.0$ & $42.1 \pm 14.9$ \\
\% change & $28.7 \pm 11.9^{* *+}$ & $38.4 \pm 15.0^{*}$ \\
4 Weeks & $-\mathbf{3 0 . 8}^{+}$ & $-\mathbf{8 . 8}$ \\
\% change & $25.0 \pm 12.2^{* *++}$ & $38.1 \pm 15.1^{* *}$ \\
6 Weeks & $-\mathbf{3 9 . 8}^{+++}$ & $-\mathbf{9 . 5}$ \\
\% change & $23.6 \pm 10.4^{* * *++}$ & $38.1 \pm 15.1^{* *}$ \\
\hline
\end{tabular}


Effects of D-002, a mixture of beeswax alcohols, on osteoarthritis symptoms...

\begin{tabular}{|c|c|c|}
\hline 8 Weeks & $20.8 \pm 10.2^{* * *++++}$ & $37.1 \pm 14.6^{* *}$ \\
\% change & $-\mathbf{4 9 . 9 ^ { + + + }}$ & $\mathbf{- 1 1 . 9}$ \\
\hline
\end{tabular}

Values are means $\pm \mathrm{SD}, \mathrm{p}<0.0001$ (ANOVA test)

$* \mathrm{p}<0.01 ; * * \mathrm{p}<0.001 ; * * * \mathrm{p}<0.0001$ versus baseline (t test for dependent samples) (Bonferroni adjustment) ${ }^{+} \mathrm{p}<0.05 ;{ }^{++} \mathrm{p}<0.01 ;{ }^{+++} \mathrm{p}<0.001{ }^{++++} \mathrm{p}<0.0001$ versus placebo (t test for independent samples)

Table 4. Changes in pain, stiffness and physical function scores by treatment group

\begin{tabular}{|c|c|c|c|c|c|c|}
\hline & \multicolumn{2}{|c|}{ Pain score } & \multicolumn{2}{|c|}{ Stiffness score } & \multicolumn{2}{|c|}{ Physical function } \\
\hline & D-002 & Placebo & D-002 & Placebo & D-002 & Placebo \\
\hline Baseline & $10.5 \pm 3.5$ & $9.9 \pm 4.0$ & $3.4 \pm 2.1$ & $3.4 \pm 1.8$ & $27.5 \pm 9.6$ & $28.7 \pm 10.8$ \\
\hline 2 Weeks & $7.5 \pm 4.4^{* *}$ & $8.8 \pm 4.2^{*}$ & $2.3 \pm 1.1^{* *_{+}}$ & $3.1 \pm 1.7$ & $18.9 \pm 7.5^{* *++}$ & $26.4 \pm 10.9^{*}$ \\
\hline$\%$ change & -28.6 & -11.1 & $-32.3+$ & -8.8 & $-31.3++$ & -8.0 \\
\hline 4 Weeks & $6.5 \pm 4.5^{* *}$ & $8.7 \pm 4.2^{*}$ & $2.0 \pm 1.1^{* *+}$ & $3.0 \pm 1.7$ & $16.5 \pm 7.8^{* *+++}$ & $26.4 \pm 10.9^{* *}$ \\
\hline$\%$ change & $-38.1^{+}$ & -12.1 & $-41.2^{+}$ & -11.8 & $-40.0^{+++}$ & -8.0 \\
\hline 6 Weeks & $6.0 \pm 4.0^{* * *+}$ & $8.7 \pm 4.2^{*}$ & $2.0 \pm 1.0^{* *+}$ & $3.0 \pm 1.7$ & $15.6 \pm 6.8^{* *+++}$ & $26.4 \pm 10.9^{* *}$ \\
\hline$\%$ change & $-42.8^{+}$ & -12.1 & $-41.2^{+}$ & - 11.8 & $-43.3^{+++}$ & -8.0 \\
\hline 8 Weeks & $5.7 \pm 4.0^{* * *++}$ & $8.4 \pm 3.6^{*}$ & $2.0 \pm 0.9^{* *+}$ & $2.9 \pm 1.6$ & $13.4 \pm 6.6^{* *+++}$ & $25.8 \pm 10.7^{* *}$ \\
\hline$\%$ change & $-45.7+$ & -15.1 & $-41.2+$ & -14.7 & $-51.3+++$ & -10.1 \\
\hline
\end{tabular}

Values are means \pm SD, $p<0.01, p<0.05$ and $p<0.0001$ for pain, stiffness and physical activity scores, respectively (ANOVA test)

$* \mathrm{p}<0.01 ; * * \mathrm{p}<0.001 ; * * * \mathrm{p}<0.0001$ versus baseline ( $\mathrm{t}$ test for dependent samples) (Bonferroni adjustment) ${ }^{+} \mathrm{p}<0.05 ;{ }^{++} \mathrm{p}<0.01 ;{ }^{+++} \mathrm{p}<0.001{ }^{++++} \mathrm{p}<0.0001$ versus placebo (t test for independent samples)

Table 5. Occurrence of adverse events (AEs) during the study

\begin{tabular}{|c|c|c|c|c|}
\hline & \multicolumn{2}{|c|}{$\mathbf{D - 0 0 2}(\mathbf{n}=\mathbf{3 0})$} & \multicolumn{2}{c|}{ Placebo $(\mathbf{n}=\mathbf{3 0})$} \\
\hline AEs & $\mathbf{n}$ & $\mathbf{\%}$ & $\mathbf{n}$ & $\mathbf{\%}$ \\
\hline Headache & 1 & 3.3 & 1 & 3.3 \\
\hline Somnolence & 1 & 3.3 & 0 & 0.0 \\
\hline Cervical pain & 0 & 0.0 & 1 & 3.3 \\
\hline Lower limb pain & 0 & 0.0 & 1 & 3.3 \\
\hline Increased appetite & 0 & 0.0 & 1 & 3.3 \\
\hline Total of AEs reported & $\mathbf{2}$ & $\mathbf{6 . 7}$ & $\mathbf{4}$ & $\mathbf{1 3 . 3}$ \\
\hline Subjects experiencing AEs & $\mathbf{2}$ & $\mathbf{6 . 7}$ & $\mathbf{3}$ & $\mathbf{1 0 . 0}$ \\
\hline
\end{tabular}

n: number of cases

No significant between group differences were found (Fisher Exact Probability test) 